\title{
Angular Momentum Transport and Mixing by Magnetic Fields
}

\author{
H.C. Spruit \\ Max-Planck Institut für Astrophysik, Postfach 1317, 85741 Garching, \\ Germany
}

\begin{abstract}
Magnetic fields can be created in stably stratified (non-convective) layers in a differentially rotating star. A magnetic instability in the toroidal field (wound up by differential rotation) replaces the role of convection in closing the field amplification loop. A dynamo model is developed from these ingredients, and applied to the problem of angular momentum transport in stellar interiors. It produces a predominantly horizontal field. The process is found to be more effective in transporting angular momentum than the known hydrodynamic mechanisms, with the possible exception of transport by internal gravity waves.
\end{abstract}

\section{Introduction}

Angular momentum transport in stars (i.e. internal torques) has so far been modeled almost exclusively with prescriptions inspired by hydrodynamical processes such as rotation-induced circulation and shear instabilities. The physical understanding of these processes under the conditions in the stably stratified interiors of stars is well developed, mostly through Jean-Paul Zahn's fundamental contributions. The application to stellar evolution, by Maeder and others, yields encouraging results for rapidly rotating massive stars, as described elsewhere in this volume. In other cases, straightforward application (i.e. without gross tuning of added parameters) still leaves large discrepancies with the observations. The slow rotation of the solar interior is one of these, another the unrealistically large rotation rates predicted for newly born neutron stars.

Magnetic fields are sensibly treated as a 'last resort', given that the relevant purely hydrodynamic processes are already somewhat intricate. The high efficiency of angular momentum transport indicated by at least some observations, however, may well point to an important contribution from magnetic torques. At the same time, the complication introduced by magnetic processes is not quite as serious as might appear at first sight. The relevant processes have been studied extensively over the past few decades, and by now are rather well understood. On the other hand, many of the complications are quite analogous to the hydrodynamic case. The stabilizing effects of thermal and compositional stratification and the effect of thermal diffusion are quite similar in both cases, for example. In the following, I summarize a theory for transport of angular momentum and mixing that builds on these insights. It has been described in more detail in Spruit(1999, 2002).

Magnetic field generation by differential rotation is usually regarded as a process operating in the convective zones of stars. Convection, or other imposed 
velocity fields like waves or shear turbulence, are not really necessary, however, for a dynamo process to operate. An example of a magnetic field produced by differential rotation without the assistance of an imposed small scale velocity field is the field produced in accretion disks (Hawley et al. 1996). In this kind of dynamo process, the differential rotation produces a small scale magnetic field on its own: the role of an imposed velocity field in generating a new the poloidal field component is replaced by an instability in the magnetic field itself. The magnetic instability operates on the toroidal field that is produced by winding up of the radial field component, distorts it, and creates new radial field components. The instability can either be of the Velikhov-Chandrasekhar-BalbusHawley type (hereafter BH instability, see Velikhov 1959; Chandrasekhar 1960; Balbus \& Hawley 1991), or a buoyancy-driven instability (Parker 1966), though the properties of the resulting field may depend on which of these instabilities is the most important.

In the same way, the generation of a magnetic field in a star requires only one essential ingredient: a sufficiently powerful differential rotation. The recreation of poloidal field components which is needed to close the 'dynamo loop' can be achieved by an instability in the toroidal field, of which there exists a variety including those (BH and buoyancy instabilities) that operate in disks. Such instabilities do not require the presence of convection, and can take place also in the stably stratified interior of a star.

The instabilities of a predominantly toroidal field in stably stratified regions in a star have been summarized from the existing literature in a previous paper (Spruit 1999, hereafter Paper I). I concluded there that the first instability likely to set in is a pinch-type instability. The essential properties of this instability in the stellar context were established by R.J. Tayler (Tayler 1973; Markey \& Tayler 1973, 1974; see also Tayler 1957; Goossens et al. 1981). What makes it of particular importance are the absence of a threshold for instability (at least in the absence of viscous damping and magnetic diffusion; more about this below), and the short growth time, of the order of the Alfvén crossing time. It can can operate under conditions where $\mathrm{BH}$ and buoyancy instabilities are suppressed by the stable stratification.

\subsection{Angular Momentum Transport}

The main interest of a magnetic field generated in the stable layers lies in its ability to exert internal torques. If such torques can operate on time scales short compared with the spindown time scale or the stellar evolution time scale, respectively (depending which of these processes is the main source of differential rotation), they can strongly affect the degree of differential rotation that can survive in a star. In some cases, they may maintain a state of nearly uniform rotation. For example, the usual interpretation of the rotation of white dwarfs and neutron stars as a remnant of the initial angular momentum of their mainsequence progenitors would require a very large degree of differential rotation between core and envelope of these stars during their evolution on the giant and AGB branches. Spruit \& Phinney (1998) have argued that, in the absence of more detailed knowledge, angular momentum processes are more likely to be either too ineffective or too effective, compared with the intermediate effectiveness needed to explain the rotation rates of white dwarfs and neutron stars. If 
the process is ineffective, leading to evolution at approximate conservation of angular momentum, the cores of stars on the AGB are very rapidly spinning, and much too rapidly rotating white dwarfs and neutron stars are the result. On the other hand, effective coupling leads to nearly uniform rotation and too slow rotation of the remnants. In the latter case, other mechanisms must contribute to the rotation of white dwarfs and neutron stars, such as birth kicks for neutron stars (Spruit \& Phinney 1998), and nonaxisymmetric mass loss on the AGB for white dwarfs (Spruit 1998).

Microscopic viscosity is negligible in stars as a source of internal torque. Hydrodynamic processes such as circulation (Zahn 1992; Maeder and Zahn 1998) and hydrodynamic instabilities are potentially important. In practice, however, the torques transmitted by most of these processes scale as $(\Omega / N)^{2}$ (where $\Omega$ is the rotation rate and $N$ the buoyancy frequency) and are most effective in rapidly rotating stars. They cannot explain the low rotation rate of the solar core (Spruit et al. 1983). An exception is angular momentum transport by internal waves generated by pressure fluctuations in a nearby convective envelope (Zahn et al. 1997), which scales as a lower power of $\Omega$. Even these waves, however, appear insufficient to explain the near-uniform rotation observed in the solar interior (Talon \& Zahn 1998; Kumar et al. 1999, see however Talon 2004).

If only these non-magnetic angular momentum transport processes are included, pre-supernova cores rotate much too fast to explain the majority of supernovae (Heger et al. 2000), though they may rotate at the right rate for the collapsar model of gamma-ray bursts (McFadyen, Woosley \& Heger 2001 and references therein). [An exception may again be the transport by internal waves, which is not yet included in standard evolution codes.]

\subsection{Magnetic Torques, the Example of the Sun}

Magnetic fields are quite effective at transporting angular momentum, even at strengths much below the values observed at the surfaces of magnetically active stars or the magnetic A stars (Mestel 1953). The Maxwell stresses transport angular momentum in the radial direction at a rate proportional to $B_{r} B_{\phi}$, and in latitude at a rate proportional to $B_{\theta} B_{\phi}$. If the observed internal rotation pattern in the solar core is due to magnetic stresses, the weak gradient in latitude compared with the radial gradient then indicates that $B_{\theta} \gg B_{r}$ in the tachocline. This fact, in combination with a sufficient amplitude of the magnetic field, are to be explained by a prospective theory for magnetic angular momentum transport that is applicable to the core of the Sun.

A differential rotation-driven dynamo operating with magnetic instabilities can do just this (Paper II). In the following the processes involved are described qualitatively. The evolution of massive stars including the torques exerted by the small-scale field generated by this process have been included in calculations by Heger (2004).

An important assumption in the following is that the initial magnetic field is sufficiently weak, so that magnetic forces can be neglected initially. Thus, I ignore the possibility that the star started with a strong magnetic field, such as those of the magnetic A-stars or the magnetic white dwarfs. The nature and origin of these is a separate issue, not fully understood at the moment. The distinction between this case and the case of an initially weak field is illustrated 


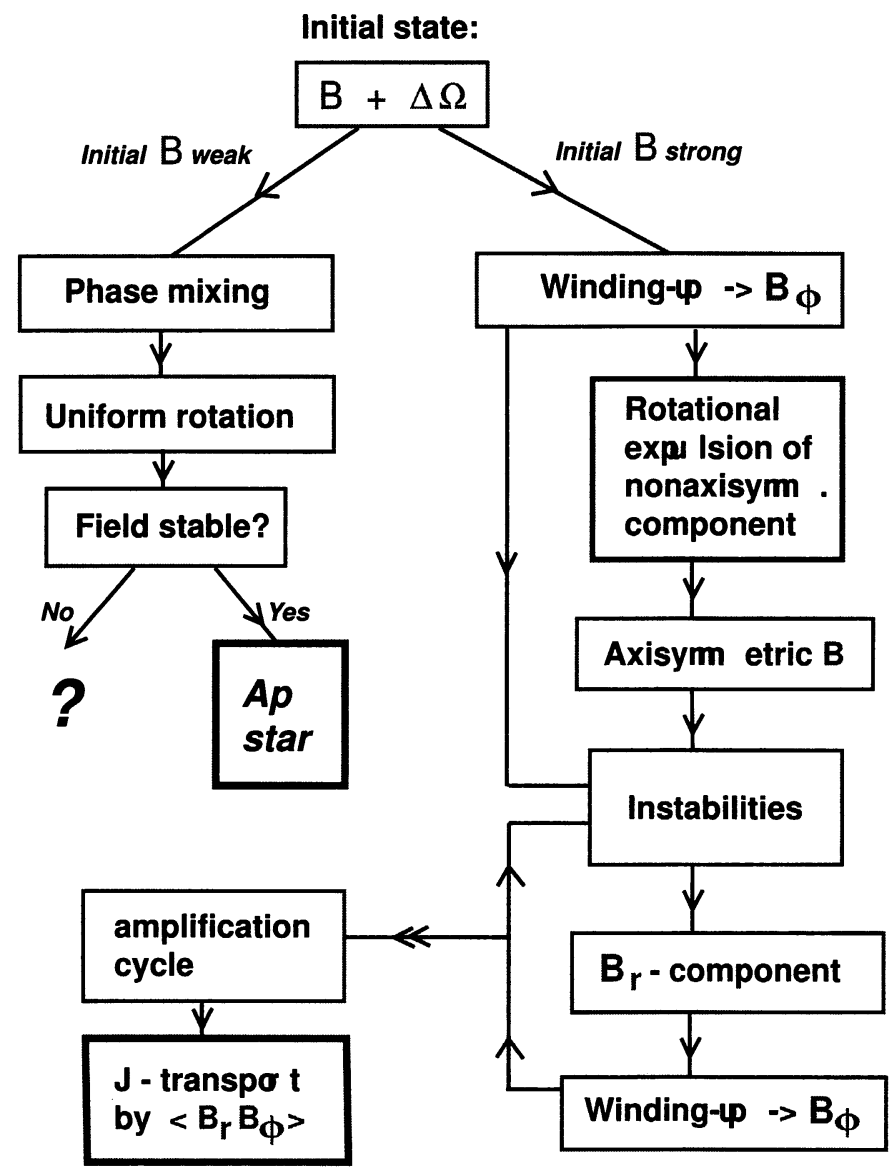

Figure 1. Flow diagram for the evolution of a magnetic field in a differentially rotating star, based on the known magnetohydrodynamic processes (cf. Paper I). The text deals mostly with case of an initially weak field. Less is known about the evolution when the star starts with a strong magnetic field (left upper left part of the diagram). 
in the flow diagram in figure 1, which summarizes the currently known MHD processes that are relevant for the evolution of magnetic fields in (radiative zones of) stars. For a discussion of some of these processes see also Charbonneau (2004).

\section{The Dynamo Process}

I assume that the star's rotation rate is a function of the radial coordinate $r$ only ('shellular' rotation, cf. Zahn 1992). This is done for convenience only.

On account of its assumed weakness, the radial component $B_{r}$ of the field is wound up by differential rotation. After only a few differential turns, the resulting field is predominantly azimuthal, $B_{\phi} \gg B_{r}$, and its strength increases linearly with time, until it becomes unstable.

\subsection{Instabilities}

As long as the magnetic field is weak, the energy to be gained from the field by any instability is small, and instability is possible only for displacements that avoid doing work against powerful restoring forces: the gas pressure and the buoyancy force. The first instability to set in (for a review of the relevant instabilities see Paper I) is a nonaxisymmetric (typically $m=1$ ) interchange in which the displacements are nearly incompressive $(\operatorname{div} \xi \ll \xi / r$, thus avoiding work against the gas pressure), and nearly horizontal (along equipotential surfaces), avoiding work against the buoyancy force.

In the absence of diffusive damping effects, such as magnetic diffusion and viscosity, an arbitrarily weak azimuthal field is unstable (Tayler 1973; Pitts \& Tayler 1986). The form of the instability is related to kink instabilities in a linear pinch. For a purely azimuthal field, the instability is a local one: unstable displacements exist as soon as a local instability condition is satisfied at any point in the star (Tayler 1973). I refer to the instability, in the form it takes in the stably stratified conditions of a stellar interior as 'Tayler instability' for short.

\subsection{Instability without Thermal Diffusion}

In this subsection, I ignore the thermal diffusion, but take into account the diffusion of the magnetic field by Ohmic resistivity. The growth rate of the instability in the absence of constraints like rotation is of the order $\sigma \sim \Omega_{\mathrm{A}}$ [where $\Omega_{\mathrm{A}}$ is the Alfven frequency]. Thus, the kinetic energy released by an unstable displacement of amplitude $\xi$ is $\frac{1}{2} \Omega_{\mathrm{A}}^{2} \xi^{2}$ (per unit of mass). This energy is supplied by the field configuration. In order to avoid wasting this energy by doing work against the stable stratification, the unstable displacements must be nearly along equipotential surfaces, $\xi_{r} \ll \xi_{\mathrm{h}}$, where $\xi_{\mathrm{h}}=\left|\left(\xi_{\theta}, \xi_{\phi}\right)\right|$ is the horizontal displacement. If $l_{r}$ and $l_{\mathrm{h}}$ are the radial and horizontal length scales of the displacement, the condition that $\xi$ be nearly incompressive implies that $l_{\mathrm{h}} / l_{r} \approx \xi_{\mathrm{h}} / \xi_{r} \gg 1$. For such displacements, the work done against the buoyancy force is (per unit mass) $\frac{1}{2} \xi^{2} N^{2}\left(l_{r} / l_{\mathrm{h}}\right)^{2}$. Hence the unstable displacements have to satisfy $l_{r} / l_{\mathrm{h}}<\Omega_{\mathrm{A}} / N$. 
In a star, the largest length scale available is of order $r$, so we must have $l_{\mathrm{h}}<r$. So the radial length scale has to satisfy

$$
l_{r}<r \Omega_{\mathrm{A}} / N
$$

On the other hand, if the radial length scale is too small, field perturbations cannot grow because the magnetic diffusivity smoothes them out too fast. The corresponding limit is found by equating the magnetic diffusion time scale $t_{\mathrm{d}}$ on the length scale $l_{r}$ to the growth time scale $\sigma^{-1}$ of the instability. In presence of a strong Coriolis force, $\Omega \gg \Omega_{\mathrm{A}}$, the intrinsic growth rate of the instability (in the absence of stratification and magnetic diffusion) is $\sigma \sim \Omega_{\mathrm{A}}^{2} / \Omega$. Hence the radial length scale has to satisfy

$$
l_{r}^{2}>\eta \Omega / \Omega_{\mathrm{A}}^{2}
$$

Combining the two limits yields

$$
\frac{\Omega_{\mathrm{A}}}{\Omega}>\left(\frac{N}{\Omega}\right)^{1 / 2}\left(\frac{\eta}{r^{2} \Omega}\right)^{1 / 4} .
$$

This is, for the case when thermal diffusion can be neglected, and up to a numerical factor of order unity, the correct instability condition as derived from Acheson's (1978) dispersion relation for azimuthal magnetic fields (Paper I).

\subsection{Effect of Thermal Diffusion}

As in the case of hydrodynamic mechanisms, thermal diffusion has a major effect by removing stabilizing buoyancy effects on small length scales. It must be included in a realistic treatment of magnetic instabilities as well.

Since heat is transported by photons, while viscosity and magnetic fields diffuse by Coulomb interactions, the Prandtl number $\operatorname{Pr}=\nu / \kappa$ and the diffusivity ratio $s=\eta / \kappa$ are very small in a stellar interior (of the order $10^{-6}-10^{-4}$ ). Here $\nu$ is the viscosity, $\kappa=16 \sigma T^{3} /\left(3 \kappa_{\mathrm{R}} \rho^{2} c_{p}\right)$ is the thermal diffusivity, and $\eta$ the magnetic diffusivity. The viscosity is of the same order but somewhat smaller than $\eta$ so that the main mechanism damping the instability is magnetic diffusion. I ignore viscous damping in the following, and also assume that gradients in composition ( $\mu$-gradients) can be neglected, so the stabilizing stratification is due entirely to the entropy gradient. In general, both have to be included; this is discussed briefly below. The effect of the $\mu$-gradients on the end-result of evolution calculations (Heger et al. 2004).

If unstable displacements take place on a length scale $l_{r}$ and time scale $\tau$, their temperature fluctuations diffuse away on the time scale $\tau_{\mathrm{T}}=l_{r}^{2} / \kappa$. On the time scale $\tau$ they are therefore reduced by a factor $f=\left(\tau / \tau_{\mathrm{T}}+1\right)$. This can be taken into account by introducing an effective thermal buoyancy frequency $N_{\mathrm{e}}$ :

$$
N_{\mathrm{e}}=N / f^{1 / 2}
$$

The stabilizing effect of the stratification is thus reduced by thermal diffusion, and instabilities take place more readily. This has apparently been realized first by Townsend (1958), who used the argument in the context of shear flows in a 
stratified atmosphere. It has been extended to the astrophysical case by Zahn (1974, 1983).

Applying the argument to the present case of Tayler instability, where the stratification also plays a dominant role, we repeat the heuristic argument in the preceding section by substituting $N_{\mathrm{e}}$ for $N$ in (4). We simplify the algebra by assuming $\tau_{\mathrm{T}} \ll \tau$ (this can be checked afterwards). The minimum radial wavenumber $k_{\mathrm{c}}$ at which the instability can take place is then

$$
(k r)_{\mathrm{c}}=\left(\frac{N}{\Omega}\right)^{1 / 2}\left(\frac{r^{2} \Omega}{\kappa}\right)^{1 / 4} .
$$

The corresponding length scale $l_{\mathrm{c}}=k_{\mathrm{c}}^{-1}$ is the largest radial length scale on which the instability can take place. Note that it is now independent of the strength of the azimuthal field, contrary to the case when thermal diffusion is neglected (eq 4). The instability condition becomes, by the same derivation as before:

$$
\frac{\Omega_{\mathrm{A}}}{\Omega}>\left(\frac{N}{\Omega}\right)^{1 / 2}\left(\frac{\kappa}{r^{2} \Omega}\right)^{1 / 4}\left(\frac{\eta}{\kappa}\right)^{1 / 2} .
$$

This is the condition derived in Paper $\mathrm{I}^{1}$. The conditions for validity are

$$
\Omega_{\mathrm{A}} \ll N \ll \Omega, \quad \eta \ll \kappa,
$$

and the buoyancy due to composition gradients has to be negligible.

The instability condition is approximate and ignores multiplying factors of order unity. More exact conditions can be derived from Acheson's dispersion relation (see Appendix in Paper I).

\subsection{Composition Gradients}

Since the diffusion of particles is much slower than that of photons, the buoyancy due to stabilizing composition gradients is not reduced on small scales. If the stabilization is dominated by the $\mu$-gradient, the instability condition reverts to (3), with the adiabatic buoyancy frequency $N$ replaced by the compositional buoyancy frequency $N_{\mu}=(g \mathrm{~d} \ln \mu / \mathrm{d} r)^{1 / 2}$. The instability is much weaker in zones with substantial $\mu$-gradients, and a large gradient in rotation rate is needed to wind up the field to amplitudes sufficient for instability.

\subsection{Field Generation}

The Field-Amplification Loop Consider the dynamo process as starting with the Tayler instability of an azimuthal field, which itself is produced by the winding-up of a seed field. The instability generates a new field component whose length scale in the radial direction is small because of the strong effect of the stratification (cf Eq. 5). This instability-generated, small scale field has zero average. The differential rotation acts on this field, winding it up into a new contribution to the azimuthal field. This again is unstable, thus closing the dynamo loop. Once the dynamo process has built up, the original seed field is

\footnotetext{
${ }^{1}$ Note that there is a typo in Eq. (49) of that paper. The last occurrence of $N$ should be an $\Omega$. The correct result is given in the appendix of that paper in Eq. (A29).
} 
unimportant. All three magnetic field components then have small length scales in the $r$-direction, but much larger scales in the horizontal directions.

The energy for the dynamo process is fed in by differential rotation only, and the small scale field is generated by instability of the azimuthal field component itself. This is fundamentally different from a dynamo driven by an imposed small scale velocity field such as convection.

The process of field generation can be broken down into two conceptual steps. In the first step, we ask ourselves at what level the Tayler instability will saturate, if the strength of the initial azimuthal field is given. This level determines the amplitude of the small scale processes generated by the instability. These in turn determine the rate of decay of a given azimuthal field by these processes.

In the second step, we ask how fast the azimuthal field is regenerated by the differential rotation. The process here is simply the stretching of the small scale magnetic field by the differential rotation. The argument is then wrapped up by requiring the regeneration rate to match the decay rate. This yields the equilibrium field strength of the dynamo process.

Analogy: Convection in a Stellar Envelope These steps can be illustrated with the analogy of stellar convection. The Rayleigh number is $R a=g H^{3}(\nabla-$ $\left.\nabla_{\mathrm{a}}\right) /(\nu \kappa)$, where $\nu$ and $\kappa$ are the viscosity and thermal diffusivity as before, and $g$ the acceleration of gravity. Instability sets in when $R a$ reaches the critical value $R a_{\mathrm{c}}$. The typical convective velocity and length scale $v_{\mathrm{c}}$ and $l_{\mathrm{c}}$ can be thought of as acting like an effective diffusivity $\nu_{\mathrm{e}} \sim v_{\mathrm{c}} l_{\mathrm{c}}$. For $R a \gg R a_{\mathrm{c}}$, we now estimate the amplitude of convection by assuming that the effective diffusivity $\nu_{\mathrm{e}} \approx \kappa_{\mathrm{e}}$ is just so large that $R a=R a_{\mathrm{c}}$, when the effective diffusivities are used in the expression for $R a$. In other words, the diffusivity becomes so large that the effective Rayleigh number is just the critical value for onset of instability. With a typical length scale $l_{\mathrm{c}}=H$, one verifies that this yields a convective energy flux of $F_{\mathrm{c}}=\rho(g H)^{3 / 2}\left(\nabla-\nabla_{\mathrm{a}}\right)^{3 / 2}$. Up to a numerical factor of order unity, this is the mixing length expression for the convective flux. This well-known 'effective Rayleigh number' argument is thus equivalent to a mixing length estimate.

While the previous argument yields the convective flux when the entropy gradient $\nabla-\nabla_{\mathrm{a}}$ is given, this flux is usually fixed by other factors. Requiring the flux to equal the value that follows from the star's luminosity then determines the value of $\nabla-\nabla_{\mathrm{a}}$, as well as the convective velocity amplitude $v_{\mathrm{c}}$.

The analogous steps can be applied to the magnetic dynamo process. In this analogy, the differential rotation plays the role of the energy flux (assumed to be imposed). The azimuthal magnetic field strength produced by winding-up drives the instability and corresponds to the entropy gradient, while the other magnetic field components produced by Tayler instability play the role of the convective velocity field.

The analysis is given in Paper II, I here summarize only the net results. The magnetic field produced has a small length scale in the radial direction. In this limit, the torques exerted by the field are local (in $r$ ), and can be expressed in terms of an effective viscosity $\nu_{\mathrm{e}}$. If the effect of $\mu$-gradients dominates, it is given by

$$
\nu_{\mathrm{e} 0}=r^{2} \Omega q^{2}\left(\frac{\Omega}{N_{\mu}}\right)^{4} .
$$


On the other hand, if $\mu$-gradients can be neglected, the result is

$$
\nu_{\mathrm{e} 1}=r^{2} \Omega\left(\frac{\Omega}{N_{\mathrm{T}}}\right)^{1 / 2}\left(\frac{\kappa}{r^{2} N_{\mathrm{T}}}\right)^{1 / 2} .
$$

In these expressions, $N_{\mu}$ is the buoyancy frequency for isothermal displacements, i.e. on sufficiently small scales that thermal diffusion is effective so only the $\mu$ gradient contributes to the buoyancy. The thermal buoyancy frequency $N_{\mathrm{T}}$ is given by $N_{\mathrm{T}}^{2}=N^{2}-N_{\mu}^{2}$, where $N$ is the total buoyancy frequency (i.e. for adiabatic displacements), and $q$ is the logarithmic differential rotation $q=$ $\mathrm{d} \ln \Omega / \mathrm{d} \ln r$.

The field strengths resulting from the process and the mixing effect associated with the field generation process are given in Paper II.

\section{Discussion}

\subsection{Comparison with Hydrodynamic Torques}

The dependence on $\Omega$ is steeper than in most purely hydrodynamic transport mechanisms. On the other hand, the scale of the effective viscosity multiplying this dependence, of the order $r^{2} \Omega q^{2}$, is much larger. Under conditions when both hydrodynamic turbulence according to Zahn's estimate and the dynamo process operate, the effective viscosity of the dynamo process turns out to be larger. This reflects the intrinsically high effectivity of Maxwell stresses at transporting angular momentum. The small mixing effect associated with the field generation process does not appear to have much effect on the compostion, in practical calculations (Heger, private communication).

The high effectiveness of the dynamo process in the absence of composition gradients removes most of the differential rotation in compositionally homogeneous layers of a star. The gradients concentrate into the inhomogeneous layers. Jumps in composition thus also tend to shield different zones somewhat from each other in terms of their rotation rates. This is the main effect that was ignored in the estimates made in Spruit and Phinney (1998); it allows evolved cores to continue rotating at significant rates for evolutionary siginificant periods of time (at least for the massive stars for which the calculations have been done so far).

The steep dependence on $\Omega$ causes a certain 'convergence': somewhat independent of other factors influencing the rotation, there will be a tendency for $\Omega / N_{\mu}$ to evolve into a limited range. This also makes the result somewhat independent of uncertainties in the theory.

Since the dynamo process produces its own 'turbulent diffusion', it enhances the decay of an initial field. Thus the process also enhances the distinction between two regimes: an initially weak field serves as a seed field but is eliminated by the action of the dynamo process, while a stronger initial field will prevent the field amplification cycle from operating altogether.

\section{References}

Acheson, D.J. 1978, Phil Trans. Roy. Soc. Lond. A289, 459 
Balbus, S.A., \& Hawley, J.F. 1991, ApJ 376, 214

Chandrasekhar, S. 1960, Proc. Nat. Acad. Sci. 46, 253

Charbonneau, P. 2004, this volume

Goossens, M., Biront, D., \& Tayler, R.J. 1981, Ap. Space Sci. 75, 521

Hawley J.F., Gammie C.F., \& Balbus, S.A. 1996, ApJ 464, 690

Heger, A., Langer, N. \& Woosley, S.E. 2000, ApJ 528, 368

Heger A., Woosley S. E., Langer, N. \& Spruit, H. C. 2004, this volume

Kumar, P., Talon, \& S., Zahn, J.-P. 1999, ApJ 520, 859

Maeder, A., \& Zahn, J.-P. 1998, A\&A 334, 1000

MacFadyen, A.I., Woosley, S.E., \& Heger, A. 2001, ApJ 550, 410

Markey, P., \& Tayler, R.J. 1973, MNRAS 163, 77

Markey, P., \& Tayler, R.J. 1974, MNRAS 168, 505

Mestel, L. 1953, MNRAS 113, 716 (remark on p. 735)

Parker, E.N. 1966, ApJ 145, 811

Oxford, Clarendon Press

Pitts, E. \& Tayler R.J. 1986, MNRAS 216, 139

Neubauer, F.M. 1983, ApJ 271, 335

Spruit, H.C. 1998, A\&A 333, 603

Spruit, H.C. 1999, A\&A 349, 189 (Paper I)

Spruit, H.C. 2002, A\&A 381, 923 (Paper II)

Spruit, H.C., \& Phinney, E.S. 1998, Nature, 393, 139

Spruit, H.C., Knobloch, E., \& Roxburgh, I.W. 1983, Nature 304, 520

Talon, S., \& Zahn, J.-P., 1998, A\&A 329, 315

Talon, S. 2004, this volume

Tayler, R.J. 1957, Proc. Phys. Soc. B 70, 31

Tayler, R.J. 1973, MNRAS 161, 365

Townsend, A.A. 1958, J. Fluid Mech. 4, 361

Velikhov, E.P. 1959, J.Exp. Theoret. Phys. (USSR), 36, 1398

Zahn, J.-P. 1974, in Stellar Instability and Evolution, eds. P. Ledoux et al., Reidel, Dordrecht, 185

Zahn, J.-P. 1983, in Astrophysical Processes in upper Main Sequence Stars, eds.

A.N. Cox et al., Geneva Observatory, Switzerland, 225

Zahn, J.-P. 1992, A\&A 265, 115

Zahn, J.-P., Talon S., \& Matias, J. 1997, A\&A 322, 320 